\title{
The Effectiveness of Hearing Aids on Tinnitus Management for Hearing-Impaired Listeners with Tinnitus
}

\author{
Yeo-ra Ha, Junghwa Bahng \\ Department of Audiology \& Speech Language Pathology, Hallym University of Graduate Studies, Seoul, Korea
}

이명이 있는 난청인의 보청기 착용으로 인한 이명재활의 효과

하 여 라·방 정 화

한림국제대학원대학교 청각언어치료학과

\begin{abstract}
Purpose: Hearing aids are often prescribed for listeners who have hearing loss with tinnitus. This study investigated the effectiveness of hearing aids when managing tinnitus using Tinnitus Magnitude Index (TMI). Methods: A total of 50 new hearing aid users with tinnitus participated as the experimental group. As the control group, 26 non-hearing aid users with tinnitus also attended. Both groups had similar audiometric thresholds, age ranges, and tinnitus duration. To measure the effectiveness of hearing aids for managing tinnitus, the TMI was assessed at a pre-hearing aid fitting and then two weeks after that fitting. In the control group, TMI was measured at the twoweek interval, but without any tinnitus management. Results: TMI scores were significantly different at the pre-hearing aid fitting and the post-hearing aid fitting for the experimental group. However, in the control group, TMI scores were not significantly changed within two weeks. Further, $86 \%$ of new hearing aid users reported a subjective benefit for their tinnitus after using their hearing aids. Conclusion: Hearing aids can be a practical option for managing tinnitus for hearing impaired listeners with tinnitus.
\end{abstract}

Key Words: Tinnitus, Hearing aids, Hearing loss, Tinnitus questionnaire.

Received: October 4, 2018 / Revised: October 15, 2018 / Accepted: October 15, 2018

Correspondence: Junghwa Bahng, Department of Audiology and Speech Pathology, Hallym University of Graduate Studies, 427 Yeoksam-ro, Gangnamgu 06197, Korea

Tel: +82-70-8680-6933 / Fax: +82-2-3453-6618 / E-mail: bahng.jh@gmail.com

\section{INTRODUCTION}

이명이란 외부의 특별한 자극 없이 소리를 느끼는 현상으로 여러 가지 원인에 의하여 발생할 수 있지만 그중에서도 중추신 경계 및 정서와 깊은 연관성을 가지고 있기 때문에 치료가 어 려울 뿐만 아니라 정상인의 $95 \%$ 이상이 살면서 한 번은 경험 할 정도로 흔한 증상이다(Snow, 2004). 국가마다 차이는 있지 만 이명은 다른 병에 비하여 세계적으로 높은 유병률을 보인다. American Tinnitus Association (2018)에서는 2011년에서 2012 년 national health and nutritional examination의 결과에서 4

(c) This is an Open Access article distributed under the terms of the Creative Commons Attribution Non-Commercial License (https://creativecommons.org/licenses/by-nc/4.0) which permits unrestricted non-commercial use, distribution, and reproduction in any medium, provided the original work is properly cited.
천5백만 명의 미국인이 이명을 겪고 있으며 그중 $67 \%$ 는 정기적 으로 이명을 경험하고 $30 \%$ 가 이명으로 인하여 삶의 큰 문제를 가지고 있다고 보고하였다. 영국이명협회(British Tinnitus Association, 2013)에서는 영국의 경우 성인의 $10 \%$ 가 이명을 가지 고 있으며, 그중 $10 \%$ 는 우울증, 스트레스, 수면 방해 등으로 인 해 심한 고통을 받고 있다고 하였다. 우리나라의 국민건강영양 조사에서는 이명 유병률은 2009 2011년 조사결과 전체 성인 의 19.7\%(Park et al., 2014), 2010 2012년의 조사결과로는 전체 성인의 21.4\%(Park \& Moon, 2014)로 이명 환자의 비율이 점차 증가하는 것으로 나타났다.

이명은 주로 난청, 소음 노출, 노화, 이독성 약물로 인한 청신 경의 비정상적인 변화로 인하여 발생한다. 또한 귀의 염증, 심혈 관 질환, 메니에르병, 종양, 청신경병증으로 인하여 발생하기도 
하지만 원인이 불분명하게 발생하기도 한다(Sarac \& Arli, 2018). 이명의 동반 증상으로는 스트레스, 불안, 수면장애와 이 명으로 인한 성가심이 있으며 이명이 만성화되는 경우 수면, 일, 집중력에 문제가 생겨 일상생활의 불편을 초래하고 삶의 질을 저하시킬 수 있다(Vesterager, 1994). 이명으로 인한 많은 어려움 으로 인하여 이명을 경험하는 사람 중 $20 \%$ 정도는 이명 치료를 위해 전문가의 도움을 받기 원한다(Jastreboff \& Hazell, 1998).

\section{이명재활의 방법}

일반적으로 이명재활을 위하여 소리치료(sound therapy), 인 지행동치료(cognitive behavioral treatment, $\mathrm{CBT}$ ), 이명 재훈 련(tinnitus retraining therapy, TRT), 보청기 착용 등을 시도한 다. 소리치료(sound therapy)는 이명재활의 가장 대표적인 방법 이며 가장 효과가 좋은 방법 중 하나라고 알려져 있다(Del Bo et al., 2011). 소리치료는 여러 소음을 이용하여 이명 소리를 차 폐함으로써 이명에 대한 환자의 인식이나 반응을 바꿔주는 방 법으로 진행된다. 이러한 이명차폐를 이용한 치료는 이명의 크기 를 줄여주고, 이명에 대한 집중을 분산시켜준다고 알려져 있다 (Hoare et al., 2014). 인지행동치료(CBT)는 1960년대에 Aaron Beck이 개발한 '인지치료(cognitive therapy)'를 기반으로 한다 (Meikle et al., 2008). 이명재활에 있어서 인지행동치료는 이명 소리 그 자체를 없애는 것이 아니라 이명으로 인한 부정적인 인 지적, 정서적, 행동적 반응들을 수정하는 데 목표를 둔다(Greimel \& Kröner-Herwig, 2011). 이명 재훈련(TRT)은 Jastreboff (1990)가 제시한 신경생리모델(neurophysiological model)에 기초를 둔 것으로 '감정계·신체반응계'와 청각계로 이어지는 악순환으로 인해 '이명으로 인한 고통'이 증가하므로 악순환의 고리를 끊기 위해 이명을 습관화(habituation)하는 이명 재훈련 방법이다. 현재 이명의 치료를 위하여 가장 널리 알려진 방법이 며 지시적 상담(directive counseling)과 소리치료를 병행한다. 이명 재훈련의 목적은 이명 자체를 없애는 것이 아니라 환자가 이명을 인식하지 않게 하기 위한 것이다.

위와 같은 다양한 치료 방법 중 보청기 착용은 보청기에 의 해 증폭된 소리로 이명 인지를 감소시켜 어느 정도 이명의 치료 효과가 있는 것으로 알려진 방법 중 하나이다(Halford \& Anderson, 1991). Surr et al.(1985)에 의하면 새로 보청기를 사용하 기 시작하는 환자의 $62 \%$ 가 이명을 가지고 있으며 이 환자 중 $50 \%$ 가량이 보청기 착용 후 이명 인지의 감소를 얻었다고 보고 하였다. 임상적인 측면에서 보청기는 난청이 동반된 이명 환자 에게 두 가지 이점이 있다. 첫째, 보청기는 듣고자 하는 외부의 소리를 잘 전달하여 귀 내부에서 들리는 이명에 대한 자각을 감소시키기 때문에 이명으로 인해 가려지는 소리와 성가신 느 낌을 줄이므로 원활한 대화가 가능하도록 도와준다(Del Bo \&
Ambrosetti, 2007). 둘째, 이명은 소리를 듣기 위한 적절한 소리 자극이 없어지면서 청신경계가 부적절하게 변화하여 발생한다 고 알려져 있으므로, 보청기를 통하여 적절한 외부 소리를 전 달함으로써, 청신경계가 비정상적으로 변화하는 것을 예방하는 효과도 있다(Kim \& Choi, 2011).

\section{이명재활 효과의 주관적 평가}

이명재활의 효과를 측정하기 위해서는 이명의 객관적인 변 화를 측정하기 어렵기 때문에 이명의 주관적 특성을 파악하여 야 한다. 이명의 주관적 특성을 살펴보기 위해서는 설문지를 사용한다. 현재 우리나라 다수의 임상에서 이명의 괴로움, 이명 의 치료 효과 평가를 위해 사용하고 있는 설문지는 Newman et al.(1996)이 개발한 Tinnitus Handicap Inventory (THI)로 본 설문지는 정서적 스트레스와 기능적 장애, 재앙화 생각의 각 항목으로 나누어서 총 25 개의 질문으로 구성되어 있고 "아니 다"는 0점, "가끔 그렇다"는 2점, "그렇다"는 4점으로 총점을 구 하는데 이와 같이 여러 개의 문항으로 인해 설문지를 작성하는 데 소요되는 시간이 길며 이명에 대한 감정과 반응이 혼재되어 있다(Schmidt et al., 2014). 또한 이러한 이명의 증상을 질문하 는 여러 설문지는 이명의 심각성에 대한 개인차를 측정하는 데 유용함은 입증되었지만, 이명의 치료 관련 변화에 있어 양적인 변화를 보도록 설계되어 있지 않다(Meikle et al., 2008).

이러한 문제점으로 인해 이명의 크기에 대한 인식을 연구해 야 한다는 요구에 맞춰 Tinnitus Magnitude Index (TMI)가 개 발되었다(Schmidt et al., 2014). 이 설문지는 일반적인 우울 증 상에 대한 정서적 반응에 의해 혼란스러워하지 않는 이명의 정 도를 측정할 수 있으며 이명의 크기, 이명의 인지시간, 이명의 심각성 등 3가지 하위 항목으로 빠르고 쉽게 작성할 수 있다는 것과 설문을 작성하는 가장 최근의 이명 상태를 평가하기 때문 에 이명으로 인한 가장 최근의 변화를 쉽게 알 수 있다는 특징 을 갖고 있다. 또한 이명에 대해 상대적으로 독립적인 이명의 크 기를 측정하는 설문지이다. 본 설문지는 Hwang \& Bahng(2018) 이 한국어로 번역하여 신뢰도를 검증하였다.

본 연구의 목적은 이명이 있는 난청인을 대상으로 한국어 $\mathrm{TMI}$ 를 이용하여 보청기가 이명의 재활에 있어서 효과적임을 증명하고자 하는 데 있다.

\section{MATERIALS AND METHODS}

\section{연구 대상}

본 연구는 2017년 9월부터 12월까지 서울과 경기도에 위치한 5 개 이비인후과에 내원하는 신규 보청기 착용 피검자 50 명을 대상으로 하였으며 과거 이명치료나 보청기 착용 경험이 없으 
며 이명이 있다고 보고한 난청인들을 대상으로 연구를 진행하 였다. 대조군으로는 이명을 경험하고 있으나 보청기 착용은 하 지 않은 26명을 대상으로 하였다. 실험군과 대조군의 청력은 Figure 1에, 이명의 정보는 Table 1에 제시하였다. 실험군으로 참 여한 보청기 착용자들은 보청기 안에 내재되어 있는 소리발생 기 등의 부가적인 성능은 사용하지 않았다. TMI 설문지는 보청 기 착용 전, 그리고 착용 2주 후에 조사하였으며 대조군도 마찬 가지로 2주 간격으로 조사하였다.

\section{이명 설문지 평가}

$\mathrm{TMI}$ 설문지는 보청기를 착용하기 전과 착용 후 2주째에 시 행하였으며 설문지를 제공하여 이명으로 인한 괴로움을 피검 자가 직접 읽고 표시하도록 하였으나 피검자가 내용을 이해 못 하거나 작성을 어려워하는 경우에는 검사자가 읽어주고 기록하 는 방법을 병행하여 진행하였다. TMI 점수는 1 번 문항은 이명 의 크기를 묻는 질문으로 0 10점, 2번 문항은 이명을 인지하는 시간을 0 100\%를 각각 10점 척도로 표시하게 하였으며, 3번 문 항은 이명의 심각성을 $100 \mathrm{~mm}$ 선 위에 스스로 심각하게 느끼 는 정도에 $\mathrm{X}$ 로 표시하게 하였다. 3 번 문항의 경우 체크 표시한 부분을 $15 \mathrm{~cm}$ 자를 이용하여 직접 자로 측정하여 판단하였다.
$\mathrm{X}$ 표가 아닌 $\bigcirc$ 로 표시한 경우 $\bigcirc$ 의 가운데를 기준으로 하였 다(Hwang \& Bahng, 2018) (Appendix).

\section{통계 분석}

통계 분석은 SPSS (version 18.0; SPSS Inc., Chicago, IL, $\mathrm{USA}$ )를 사용하였다. TMI 3 문항의 척도가 모두 달라 TMI의 질문 1 번은 크기를 $0,-10$ 점을 10 점 척도로 체크하는 것으로 기 입 시 한 척도당 $\times 10$ 으로 계산하였다. TMI의 3 번의 결과는 피 검자가 표시한 부분을 $\mathrm{mm}$ 단위로 측정하여 기입하였다. 보청 기 착용 전후 $\mathrm{TMI}$ 점수 변화를 보기 위해 Wilcoxon signed rank test를 사용하였으며 보청기 착용 형태에 따른 TMI 점수 변화를 보기 위해 Mann Whiteny U test를 사용하였다. 그리고 보청기 착용 전후 TMI 점수 상관관계를 확인하기 위해 Spearman correlation test를 분석하였다.

\section{RESULTS}

\section{보청기 착용 후 주관적 설문지 점수 비교}

보청기 착용자 그룹인 실험군의 보청기 착용 전후의 TMI 점 수를 비교해 보았을 때 이명의 크기는 $5.01 \pm 2.50$ 에서 $3.86 \pm$
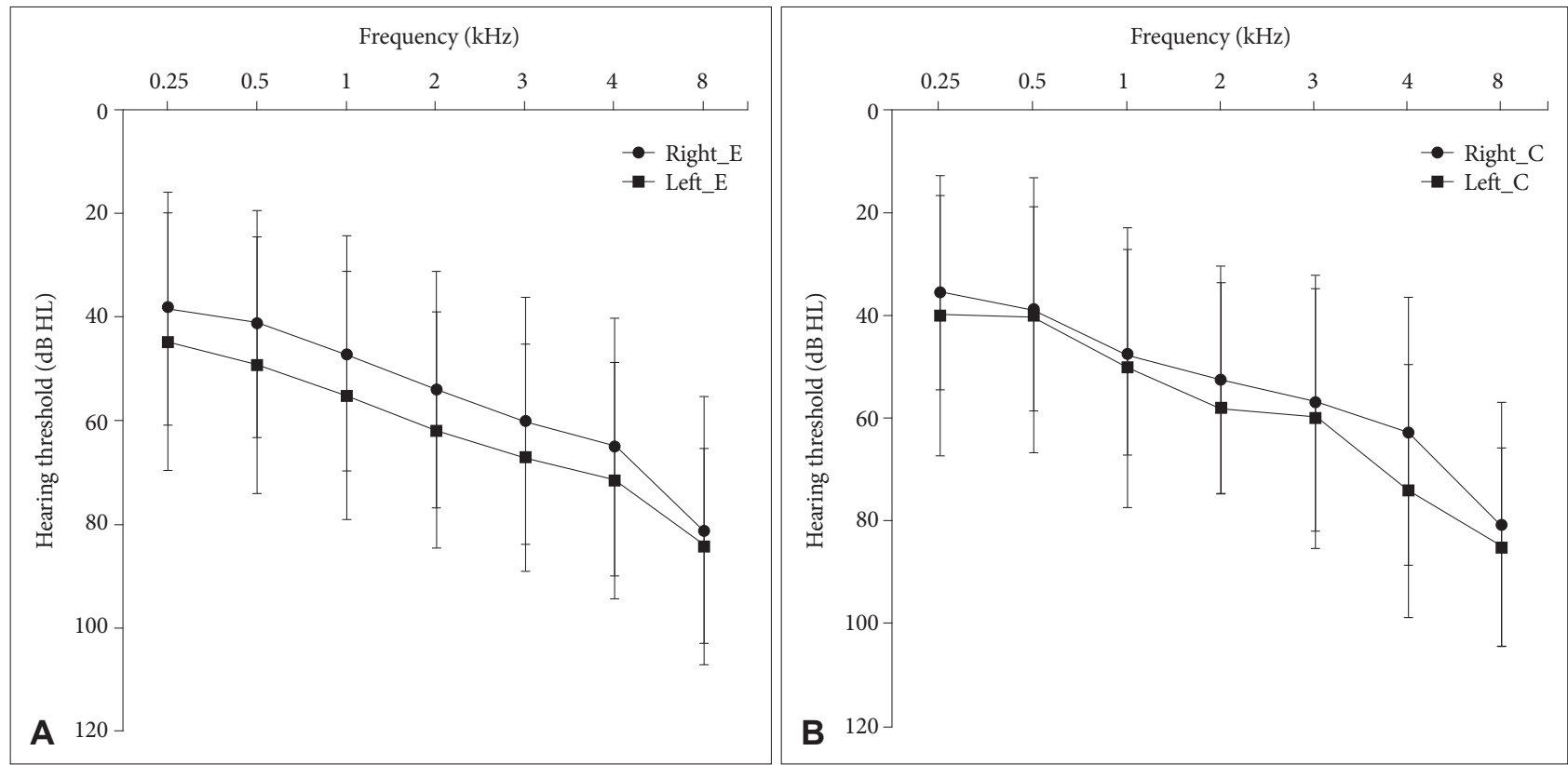

Figure 1. Mean of puretone threshold for participants (A: The experimental group. B: The control group). Each error bar means one standard deviation. Right_E: right ear experimental group, Left_E: left ear experimental group, Right_C: right ear control group, Left_C: left ear control group.

Table 1. General information of the groups

\begin{tabular}{lcccc} 
Group & Age (years) & Gender (male;female) & $\begin{array}{c}\text { Duration of tinnitus } \\
\text { (months) }\end{array}$ & $\begin{array}{c}\text { Direction of tinnitus } \\
\text { (unilateral;bilateral) }\end{array}$ \\
\hline group (hearing aid users) & $29-83$ & $19 ; 31$ & $12-240$ & $36 ; 21$ \\
(non hearing aid users) & $23-68$ & $16 ; 10$ & $19-210$ & $6 ; 20$ \\
\hline
\end{tabular}




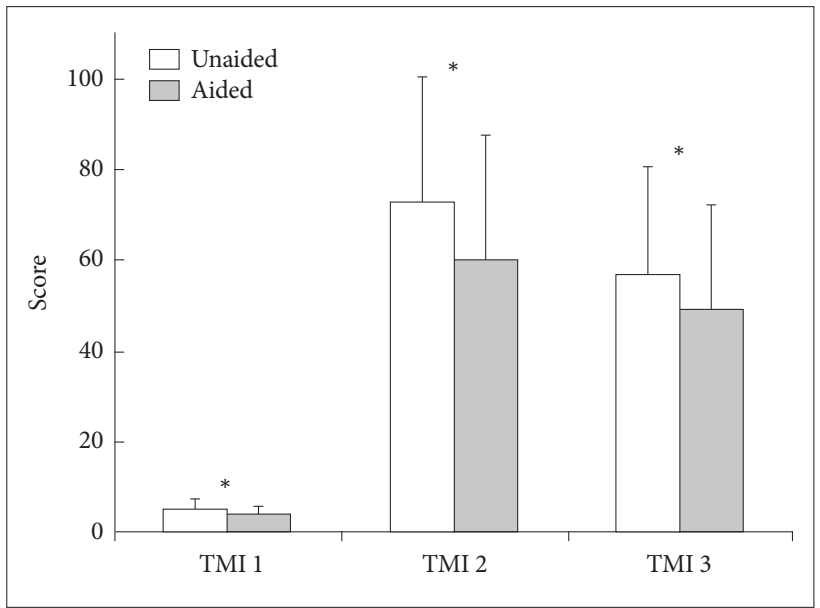

Figure 2. Comparison of the TMI scores between unaided and aided condition $\left({ }^{*} p<0.01\right)$. TMI: tinnitus magnitude index, TMI 1: TMI 1st item, TMI 2: TMI 2nd item, TMI 3: TMI 3rd item.

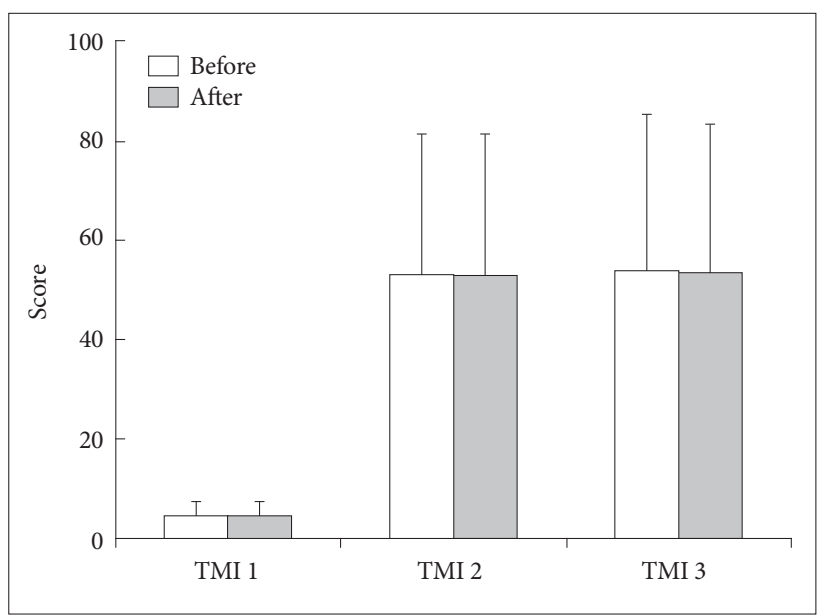

Figure 3. Comparison of the TMI scores with no treatment at twoweek interval in the control group. TMI: tinnitus magnitude index, TMI 1: TMI 1st item, TMI 2: TMI 2nd item, TMI 3: TMI 3rd item.

2.14로, 이명의 인지는 $73.1 \pm 27.16$ 에서 $60 \pm 27.53$ 으로, 이명의 심각성은 $57 \pm 23.86$ 에서 $48.1 \pm 23.75$ 의 결과를 보였으며 전체 적으로 감소하였고 통계적으로 유의하였다 $(p<0.01)$ (Figure 2).

대조군의 TMI 점수를 비교해 보았을 때 이명의 크기는 4.92 \pm 2.4 에서 $4.8 \pm 2.5$ 로, 이명의 인지는 $53 \pm 28.3$ 에서 $52.8 \pm$ 28.5 로, 이명의 심각성은 $53 \pm 31.6$ 에서 $53 \pm 30$ 의 결과를 보였 으며 3가지 항목 모두 2주 전후 결과가 통계적으로 유의하지 않 았다(Figure 3).

\section{DISCUSSIONS}

이명의 심각성은 피검자가 얼마나 또는 얼마만큼 자주 이명 으로 인한 괴로움을 느끼는지, 삶의 질을 떨어뜨리는지, 또 이 명으로 인해 어느 정도의 장애를 느끼는지 등으로 평가되며 난
청을 동반한 이명은 거의 대부분 완치가 어려운 경우가 많아 이러한 상황에서 이명의 치료 방향은 이명으로 인한 괴로움이 나 불편함을 감소시키는 것을 목적으로 한다(Folmer et al., 2004). 이명의 치료에 있어 보청기의 효과는 첫째, 이명에 대한 인식을 줄이면서 이명 자체를 차폐하는 것이고 둘째, 이명에 의 해 차폐된 소리나 음성에 의한 불쾌한 감정을 감소시켜 주는 것이다(Sheldrake \& Jastreboff, 2004).

본 연구의 목적인 보청기가 이명의 재활에서 효과적인가에 대한 질문은 연구의 결과를 살펴볼 때 보청기는 이명의 크기 인지를 감소시키는 데 효과적이라고 할 수 있다. 본 연구의 결 과에서 보청기를 사용한 후 이명의 호전은 $90 \%$ 의 피검자에서 나타났으며 각 항목으로 나누어 비교하였을 때 3 가지 모든 문 항에서 호전된 피검자를 보청기가 이명치료에 효과가 있었다고 볼 때 $86 \%$ 의 피검자에서 보청기가 이명재활에서 효과적이라고 할 수 있다. 이와는 반대로 보청기를 착용하지 않은 대조군에 서는 $34 \%$ 의 피검자에서 이명이 감소되었으며 $23 \%$ 의 피검자 에서는 오히려 불편함이 더 커진 것으로 나타났다. 이는 기존의 보고보다는 높은 수준의 비율이다. Surr et al.(1999)의 연구에 서는 46.2 54.6\%의 피검자가 보청기를 사용한 후 이명이 개선 되었다고 보고하였고, Lee et al.(2008)의 연구에서는 피검자의 $61.1 \%$ 에서 보청기로 인하여 이명이 어느 정도 이상의 도움을 받았다고 하였다. 그러나 Lee et al.(2008)이 발표한 61.1\%는 $\mathrm{THI}$ 점수가 1점이라도 호전된 경우까지 모두 포함한 것으로, 10점 이상 호전된 경우는 22.2\% 정도로 Surr et al.(1985)의 연 구나 본 연구에서의 수치보다는 낮은 비율의 피검자가 보청기 가 효과가 있다고 보고하였다. 이처럼 보청기로 이명의 감소 효 과를 보이는 비율이 차이가 있는 것은 재활의 정도를 확인하는 설문지의 차이, 효과를 측정했던 시기의 상이함 등으로 인한 것 으로 추측할 수 있다.

본 연구에 참여한 보청기 착용자들 중 설문지 3 가지 항목에 서 1가지 항목도 나아지지 않았던 대상자는 7명(14\%)이었다. 본 연구는 보청기를 처음 시도하는 난청과 이명을 가지고 있는 대상자가 참가하여 2주라는 짧은 시간 동안 이명의 변화에 대 하여 질문하였다. 만약 좀 더 긴 시간 보청기 착용 후 이명의 변 화를 볼 경우 다른 결과를 얻을 수 있으리라 예상할 수 있다. 하지만 보청기를 착용하지 않은 대조군과 비교하여 봤을 때 보 청기를 착용했던 군이 유의하게 이명의 인지, 크기, 심각성에서 모두 보청기 착용 전보다 나아졌다고 답하여 보청기를 사용하 는 것이 이명재활에 효과적임을 알 수 있다. Searchfield et al. (2010)의 연구에서는 이명재활에 있어서 난청과 이명이 모두 있 지만 보청기를 사용하는 군과 그렇지 않은 군을 나누어 이명 관련 상담을 진행하여 보청기가 이명의 효과적인 재활 방법임 을 입증하고자 하였다. 이명을 가지고 있고 비슷한 청력을 가진 
두 그룹 중 58명은 보청기를 사용하였고 29명은 보청기를 사용 하지 않았다. 두 그룹 모두 12 개월 동안 이명에 대한 상담을 진 행하고 그 이후 Tinnitus Handicap Questionnaire의 결과를 분 석한 결과 보청기를 사용하는 집단이 기능적 핸디캡(우울, 긴장, 화, 불면, 안정 등)과 사회적 핸디캡(사회생활, 과제 집중력 등) 모두 호전되었음을 보고하였다. 위의 연구결과를 비추어 보면 보청기 사용과 함께 이명재활의 상담을 함께 진행한다면 이명 완화에 효과가 있을 것으로 기대해 볼 수 있다. 이명치료에 있 어 최적의 결과는 기술적으로 진보된 보청기의 사용뿐만 아니 라 개인의 요구와 상담을 통해 얻을 수 있다. 각 피검자는 치료 계획 및 후속 조치를 통해 듣고, 상담하고, 정보를 제공받아야 하고 또 정보를 제공해야 한다. 대부분의 이명재활 과정에 상 담 세션이 포함되어 있으나 상담 그 자체만으로도 하나의 재활 로서 역할을 할 수 있다. Lee et al.(2004)은 이명으로 어려움을 겪는 피검자들을 대상으로 다른 치료는 없이 상담만을 실시하 여 이명재활의 효과를 분석하였는데, 상담 전 $77 \%$ 였던 이명 인 지 정도가 상담 실시 3 6개월 후에는 53\%로 감소하였고, 이명 의 주관적 크기와 이명에 의한 괴로움 정도 및 이명이 생활에 미치는 영향의 정도가 통계적으로 유의한 수준으로 감소하였음 을 보여주었다. 이러한 연구결과는 상담이 이명재활의 중요한 요소일 뿐 아니라 상담만으로도 재활의 효과가 나타날 수 있음 을 보여준다. 보청기와 함께 적절한 상담이 진행된다면 이명재 활에 조금 더 효과를 볼 수 있을 것으로 본다.

본 연구에서는 이명재활의 효과를 검증하기 위하여 새롭게 개발된 TMI 설문을 사용하였다. 앞서 언급한 바와 같이 TMI 의 특징은 매우 짧은 시간 내에 이명에 대한 인지시간, 크기, 심 각성에 대하여 측정할 수 있다는 큰 장점이 있다. 비록 본 연구 가 선행연구들에 비해 2주라는 매우 짧은 시간 추적관찰을 시 행하였지만, TMI의 사용을 통하여 최근의 이명 변화를 효과적 으로 측정할 수 있어 바쁜 임상에서는 매우 적절한 도구로 볼 수 있다. 본 연구의 결과에서도 보청기를 통하여 이명의 재활의 변화 정도를 쉽게 나타내었다. 그러나 본 설문지는 이명에 대한 크기를 세 문항으로만 질문하여 이명의 크기 인지 변화를 보는 설문지이기 때문에 이명에 대한 특정한 영역에서의 변화를 보 고자 하는 데에는 한계점이 있다. 즉, 이명의 크기에 대한 인지 가 호전되었다는 결과가 나오더라도 수면의 질이 나아진 것인지, 우울감이 줄어든 것인지의 판단은 어렵다. 그러므로 본 TMI 설 문지와 함께 이명으로 인하여 특정적인 변화를 측정할 목적이 있다면 다른 설문지를 상호보완해서 사용하기를 권장한다.

본 연구에서는 보청기가 이명의 재활에 있어서 효과적임을 증명하였다. 또한 새롭게 개발된 세 문항의 짧은 설문지를 통해 서도 이명의 재활 정도를 확인할 수 있었다. 비록 본 연구에서 는 이명재활의 상담을 진행하지 않아 적극적 이명의 재활 효과
를 측정할 수 없었지만 임상에서는 이명이 있는 난청인에게 적 용할 수 있으리라 본다.

중심 단어 : 이명·보청기·청력손실·이명 설문지.

\section{Ethical Statement}

The entire procedure including participant recruitment, testing compensation, and data handling followed the protocol approved by the Institutional Review Board of the Hallym University of Graduate Studies (\#HU GSAUD 697128).

\section{Acknowledgments}

The authors thank to the participants.

\section{Declaration of Conflicting Interests}

There are no conflict interests.

\section{Funding}

This work was supported by research development fund of Hallym University of Graduate Studies.

\section{REFERENCES}

American Tinnitus Association. (2018, September 20). Demographics. American Tinnitus Association. Retrieved from https:/www.ata.org/ understanding-facts/demographics.

Beck, J. S. (2011). Cognitive Behavior Therapy: Basics and Beyond. (2nd ed.), (pp.19-20). New York, NY: Guilford Press.

British Tinnitus Association. (2013, March 20). All about Tinnitus. British Tinnitus Association. Retrieved from https://www.tinnitus.org.uk/allabout-tinnitus.

Del Bo, L. \& Ambrosetti, U. (2007). Hearing aids for the treatment of tinnitus. Progress in Brain Research, 166, 341-345.

Del Bo, L., Baracca, G., Forti, S., \& Norena, A. (2011) Sound stimulation. In Møller, A. R., Langguth, B., De Ridder, D., \& Kleinjung, T. Textbook of Tinnitus (pp. 597-604). New York, NY: Springer Science \& Bussiness Media.

Folmer, R. L., Martin, W. H., \& Shi, Y. (2004). Tinnitus: Questions to reveal the cause, answers to provide relief. The Journal of Family Practice, 53(7), 532-540.

Greimel, K. V. \& Kröner-Herwig, B. (2011). Cognitive behavioral treatment (CBT). In Møller, A. R., Langguth, B., De Ridder, D., \& Kleinjung, T. (1st ed.). Textbook of Tinnitus (pp. 557-561). New York, NY: SpringerVerlag New York Inc.

Halford, J. B. S. \& Anderson, S. D. (1991). Anxiety and depression in tinnitus sufferers. Journal of Psychosomatic Research, 35(4-5), 383-390.

Hoare, D. J., Edmondson-Jones, M., Sereda, M., Akeyroyd M. A., \& Hall, D. (2014). Amplification with hearing aids for patients with tinnitus and co-existing hearing loss. Cochrane Database of Systematic Reviews, 1 , 1-31.

Hwang, D. H. \& Bahng, J. (2018). A Korean adaptation of the Tinnitus Magnitude Index: Validity and reliability. Audiology and Speech Research, 14(2), 90-99.

Jastreboff, P. J. (1990). Phantom auditory perception (tinnitus): Mechanisms of generation and perception. Neuroscience Research, 8(4), 221254.

Jastreboff, P. J. \& Hazell, J. W. P. (1998). Treatment of tinnitus based on a neurophysiological model. In Vernon J. A. Tinnitus: Treatment and Relief (pp. 201-217). Needham Heights, MA: Allyn \& Bacon.

Kim, H. Y., Choi, Y. J., Kim, D. H., Lim, H. K., Kim, G. H., Choi, M. K., et al. (2017). The association of socioeconomic status and subjective dizziness in elderly Koreans: A cross sectional study from the Korean National Health and Nutrition Examination Survey 2010-2012. Korean 
Journal of Family Practice, 7(4), 465-469.

Kim, S. C. \& Choi, J. Y. (2011). Management of presbytinnitus. Journal of the Korean Medical Association, 54(9), 935-940.

Lee, H. K., Kim, C. W., Chung, M. H., \& Kim, H. N. (2004). The effectiveness of the directive counseling in tinnitus retraining therapy. Korean Journal of Otolaryngology-Head and Neck Surgery, 47(3), 217-221.

Lee, Y. C., Byun, J. Y., Shin, I. H., \& Park, M. S. (2008). The effectiveness of the hearing aid in hearing loss with tinnitus. Korean Journal of Otolaryngology-Head and Neck Surgery, 51(11), 967-972.

Meikle, M. B., Stewart, B. J., Griest, S. E., \& Henry, J. A. (2008). Tinnitus outcomes assessment. Trends in Amplification, 12(3), 223-235.

Newman, C. W., Jacobson, G. P., \& Spitzer, J. B. (1996). Development of the Tinnitus Handicap Inventory. Archives of Otolaryngology-Head and Neck Surgery, 122(2), 143-148.

Park, K. H., Lee, S. H., Koo, J. W., Park, H. Y., Lee, K. Y., Choi, Y. S., et al. (2014). Prevalence and associated factors of tinnitus: Data from the Korean National Health and Nutrition Examination Survey 2009-2011. Journal of Epidemiology, 24(5), 417-426.

Park, R. J. \& Moon, J. D. (2014). Prevalence and risk factors of tinnitus: The Korean National Health and Nutrition Examination Survey 2010-2011, a cross-sectional study. Clinical Otolaryngology, 39(2), 89-94.

Sarac, E. T. \& Arli, C. (2018). Effects of tinnitus masking therapy in tinnitus patients with and without hearing loss. Annals of Medical Research,
25(2), 222-224.

Schmidt, C. J., Kerns, R. D., Griest, S., Theodoroff, S. M., Pietrzak, R. H., \& Henry, J. A. (2014). Toward development of a tinnitus magnitude index. Ear and Hearing, 35(4), 476-484.

Searchfield, G. D., Magnusson, J., Shakes, G., Biesinger, E., \& Kong, O. (2010). Counseling and psycho-education for tinnitus management. In Møller, A. R., Langguth, B., De Ridder, D., \& Kleinjung, T. Textbook of Tinnitus (pp. 535-556). London: Springer Dordrecht Heidelberg.

Sheldrake, J. B. \& Jastreboff, M. M. (2004). Role of hearing aids in management of tinnitus. In Snow, J. B. Tinnitus: Theory and Management (pp. 310-313. Hamilton, ON: BC Decker.

Snow, J. B. (2004). Tinnitus: Theory and Management. (pp. 1-39). Hamilton, ON: BC Decker.

Surr, R. K., Kolb, J. A., Cord, M. T., \& Garrus, N. P. (1999). Tinnitus Handicap Inventory (THI) as a hearing aid outcome measure. Journal of the American Academy of Audiology, 10(9), 489-495.

Surr, R. K., Montgomery, A. A., \& Mueller, H. G. (1985). Effect of amplification on tinnitus among new hearing aid users. Ear and Hearing, 6(2), 71-75.

Vesterager, V. (1994). Combined psychological and prosthetic management of tinnitus: A cross-sectional study of patients with severe tinnitus. British Journal of Audiology, 28(1), 1-11. 


\section{APPENDIX}

Tinnitus Magnitude Index (TMI) 설문지

아래의 각 질문을 자세히 읽어주시기 바랍니다. 각 질문을 읽고 해당하는 하나의 숫자에 동그라미 쳐주세요(예: (10\%) 또는 (1).

1. 지난 한 주 동안, 이명이 얼마나 크게 들리셨습니까?

전혀 크지 않음

매우 크게 들림

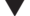

1

$2 \quad 3 \quad 4$

5

6

7

89

10

2. 지난 한 주 동안, 깨어 있는 시간 중에 이명을 인지하는 시간은 몇 \% 정도 되었습니까?

전혀 들리지 않음

항상 들림

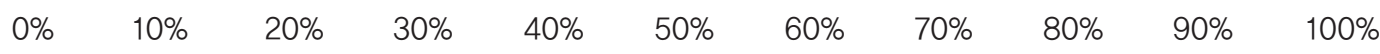

3. 지난 한 주 동안, 이명이 얼마나 심각하게 느껴졌습니까? 0부터 100 사이 중, 해당하는 곳에 X 표시 해주세요.

전혀 심각하지 않음

매우 심각함

0

100 